\title{
Poly-L-gamma-glutamic acid production by recombinant Bacillus subtilis without pgsA gene
}

\author{
Kazuhisa Sawada ${ }^{*}$, Hiroyuki Araki ${ }^{2}$, Yasushi Takimura' ${ }^{1}$, Kenta Masuda' ${ }^{1}$, Yasushi Kageyama ${ }^{1}$, Katsuya Ozaki ${ }^{1}$ \\ and Hiroshi Hagihara ${ }^{3}$
}

\begin{abstract}
Poly-gamma-glutamic acid (PGA) is a promising bio-based polymer that shares many functions with poly (acrylic acid) and its derivatives. Thus, technologies for efficient production and molecular size control of PGA are required to expand the application of this useful biopolymer. In Bacillus strains, PGA is synthesized by the PgsBCA protein complex, which is encoded by the pgsBCA gene operon, otherwise is known as yws $C$ and ywt $A B$ operons and/or cap $B C A$ operon. Hence, we investigated responsible components of the PgsBCA complex in B. subtilis for over-production of PGA. In particular, we constructed genomic pgsBCA gene-deletion mutants of B. subtilis. And also, we assembled high copy-number plasmids harboring $\sigma A$-dependent promoter, leading to high-level expression of all combinations of $p g s B C A$, pgs $B C$, pgs $B A$, pgs $C A$, pgs $B$, pgs $C$, and/or pgs $A$ genes. Subsequently, PGA production of the transformed $B$. subtilis mutant was determined in batch fermentation using medium supplemented with L-glutamate. PGA production by the transformants introduced with pgs $B C$ genes (lacking the genomic pgs $B C A$ genes) was $26.0 \pm 3.0 \mathrm{~g} \mathrm{~L}^{-1}$, and the enantiomeric ratio of $\mathrm{D}$ - and L-glutamic acid (D/L-ratio) in the produced PGA was 5/95. In contrast, D/L-ratio of produced PGA by the transformants introduced with pgsBCA genes (control strains) was 75/25. In conclusion, B. subtilis without pgsA gene could over-produce PGA with an L-rich enantiomeric ratio.
\end{abstract}

Keywords: Poly-gamma-glutamic acid, Bacillus subtilis, PgsBCA complex, Recombinant strain, L-PGA

\section{Introduction}

Poly- $\gamma$-glutamic acid (PGA) is a biopolymer comprising linear amide linkages of glutamate between $\alpha$-amino and $\gamma$-carboxyl groups (Ashiuchi and Misono 2005). PGA is the predominant source of stickiness in the Japanese traditional food Natto, which is made by fermenting steamed soybeans using Bacillus subtilis var. natto (Saito et al. 1974). PGA has a wide range of applications, because it is edible, biocompatible, biodegradable, and can be produced from sustainable materials (Luo et al. 2016). Accordingly, PGA and its derivatives are used as food additives, cosmetic materials, health-care materials, drug carriers, flocculating agents, and soil conditioners

\footnotetext{
*Correspondence: sawada.kazuhisa@kao.com

${ }^{1}$ Global R\&D-Biological Science Research, Kao Corporation, 2606

Akabane, Ichikai, Haga, Tochigi 321-3497, Japan

Full list of author information is available at the end of the article
}

(Buescher and Margaritis 2007; Luo et al. 2016). Thus, improved supply of low-cost PGA can expand the use of this bio-material.

Ashiuchi and Misono previously suggested that PGA production may be achieved using Gram-positive Bacilli such as B. anthracis and the extremely halophilic archaeon Natrialba aegyptiaca. These microbes produce PGA polymers of 10 to more than $1000 \mathrm{kDa}$ (kilo Dalton) with specific D/L-ratios (Ashiuchi and Misono 2002). Specifically, D-PGA homopolymers comprise only D-glutamate, whereas L-PGA homopolymers comprise only L-glutamate, and DL-PGA copolymers contain both enantiomers of glutamate. Among these, homopolymers have strong resistance to phagocytosis (Makino et al. 1989 ) and can act as protein stabilizers (Yamasaki et al. 2010).

PGA is synthesized in Bacillus strains by an ATPdependent transmembrane enzyme complex that 
catalyzes amide ligation (Ashiuchi et al. 2001). This complex comprises PgsBCA proteins in B. subtilis NBRC 3336 (Ashiuchi et al. 1999), YwsC and YwtAB in B. subtilis Marburg 168 (Urushibata et al. 2002), and CapBCA in B. anthracis TE702 (Makino et al. 1989). Previous studies also indicate that PgsB possesses glutamate-dependent ATPase activity, whereas PgsC has a hydrophobic structure in its transmembrane domain and facilitates PGA excretion (Ashiuchi et al. 2001). PgsA have been classified as A1-type anchor proteins and located on outer cell surface (Leenhouts et al. 1999; Narita et al. 2006).

In a previous study, Ashiuchi et al. reported that glutamate-dependent ATPase activities only in Pgs$\mathrm{BCA}$ and PgsBC complexes, and showed that PgsBCA is about 3-fold more active than PgsBC (Ashiuchi et al. 2001). These investigators also suggested that PgsB and $\mathrm{Pgs} C$ form a tight association, whereas only week interactions were observed between PgsBC and PgsA. Moreover, their experiments with D-xylose-inducible expression vectors showed no PGA production in the absence of the pgsA gene in mutant B. subtilis ISW 1214 cells, suggesting that all components of the PgsBCA complex are essential for PGA production (Ashiuchi et al. 2006).

In a contrasting study, Urushibata et al. showed that purified histidine-tagged YwsC (homolog of PgsB) synthesized PGA alone in vitro system (Urushibata et al. 2002). In addition, PGA production was observed in the absence of YwtB (homolog of PgsA), although this was much less than in wild type cells. Hence, YwtB may be necessary for maximal PGA production.

Given the discrepancies between previous studies, it remains unclear which components of PgsBCA are required for efficient PGA production, and which could be exploited for high yield commercial PGA production. To address these issues, we investigated key components of the PGA synthetic machinery by constructing vectors, which assembled using a high copy-number plasmid vector and the $\sigma \mathrm{A}$-dependent promoter from the egl-S237 gene (Hakamada et al. 2000). These molecular approaches reportedly achieved constitutive gene expression in B. subtilis during the log-growth phase (Manabe et al. 2011). Thus, we constructed mutant Bacillus subtilis strains by transforming with various combinations of $p g s B C A$ in vectors that allowed high-level expression of genes. Following transformation by the $p g s B C$ geneexpression vectors without $p g s A$ gene, recombinant $B$. subtilis produced the highest yield of PGA, as reported previously (Luo et al. 2016), and the ensuing product had increased L-glutamic acid contents. These results demonstrate a procedure for over-production of the biopolymer
L-PGA using recombinant B. subtilis having no pgsA gene.

\section{Materials and methods \\ Bacterial strains and plasmids}

Bacillus subtilis Marburg 168 was used as parental strain for construction of pgs-gene deletion mutants. The alkaliphilic laboratory strain Bacillus sp. KSM-S237 (FERM BP-7875) produces alkaline cellulase and was used as a gene resource for the promoter region of the egl-S237. The laboratory strain Bacillus sp. KSM-366 (FERM BP-6262) produces alkaline cellulase and PGA, and was used as a source of heterologous pgs-genes. Bacillus amyloliquefaciens NBRC 3022 (Hara et al. 1992) and Bacillus licheniformis ATCC 9945a (Potter et al. 2001) were also used as gene sources of PGA producers. Oceanobacillus iheyensis JCM 11309 (Takami et al. 2002) was used as a source of genes with low homology to those from B. subtilis. Escherichia coli HB101 and the plasmid pHY300PLK were purchased from Takara Bio Inc. (Kyoto, JPN). The plasmids pC194 (Ehrlich 1977) and pHYS237 (Manabe et al. 2011) were used as sources of the chloramphenicol-resistant gene $(\mathrm{Cmr})$ and as templates for cloning of plasmid fragments with egl-S237 promoter region.

\section{Bacterial growth media}

PGA producing cells were pre-cultured in Luria-Bertani (LB) medium, and were then cultured in $2 \mathrm{xL} / \mathrm{Mal}$ medium containing $2 \%(\mathrm{w} / \mathrm{v})$ tryptone (Difco), $1 \%(\mathrm{w} / \mathrm{v})$ yeast extract (Difco), $1 \%(\mathrm{w} / \mathrm{v}) \mathrm{NaCl}, 7.5 \%(\mathrm{w} / \mathrm{v})$ maltose monohydrate, and 7.5-ppm $\mathrm{MnSO}_{4} \cdot 4-5 \mathrm{H}_{2} \mathrm{O}$ or in $2 \mathrm{xL} /$ Mal+E8 medium, which was supplemented with $8 \%$ (w/v) mono-sodium glutamate monohydrate. All media were supplemented with 15-ppm tetracycline for culture of transformants. B. subtilis and E. coli strains were transformed as described previously (Manabe et al. 2011). B. subtilis transformants were selected on LB agar plates containing 10-ppm chloramphenicol (LBCm), and were regenerated on DM3 media containing 30-ppm tetracycline (DM3Tc) (Chang and Cohen 1979). E. coli transformants were selected on LB agar plates supplemented with 15-ppm tetracycline (LBTc). All components of culture media were purchased from Wako Pure Chemicals (Osaka, JPN). Antibiotics for selection media were purchased from Sigma-Aldrich (St. Louis, MO, USA).

\section{General DNA manipulations}

Restriction enzymes, DNA ligase, and DNA polymerase were purchased from Takara Bio Inc. (Kyoto, JPN). Expression vectors for heterologous pgs-genes were constructed using In-Fusion Cloning Kits (Takara Bio USA, Inc., California, USA). Genomic DNA was prepared 
according to previously described procedures (Saito and Miura 1963). PCR products and plasmid samples were purified using High Pure PCR Product Purification Kits and High Pure Plasmid Isolation Kits (Roche diagnostics, Basel, CHE). DNA sequences were analyzed using the Big-Dye Terminator v3.1 Cycle Sequencing Kits (Thermo Fisher Scientific, Massachusetts, USA) and Applied Biosystems 3130xl Genetic Analyzers (Thermo Fisher Scientific, Massachusetts, USA). DNA and protein sequences were searched using GENETYX program ver. 13 (GENETYX, Co., Tokyo, JPN).

\section{Mutant strain construction}

To construct $B$. subtilis mutant $\triangle 3$, DNA fragments for deletion of pgsBCA were amplified using PCR with the primer pairs of BCA-Dw Fw and BCA-Dw Rv (BCA-Dw fragment), $\mathrm{Cmr} \mathrm{Fw}$ and $\mathrm{Cmr} \mathrm{Rv}$ (Cmr fragment), and BCA-Up Fw and BCA-Up Rv (BCA-Up fragment). The resulting fragments contained overlapping sequences as shown in Table 1, and were ligated using the spliced overlapping-extension (SOE)-PCR method (Horton et al. 1989) with the primer pair of BCA-Dw Fw and BCAUp Rv. Competent B. subtilis cells were exposed to the resulting ligated fragment (approximately $2.9 \mathrm{~kb}$ ) using the Spizizen protocol (Young and Spizizen 1963) and the transformants were selected on $\mathrm{LBCm}$. Gene replacement efficiency was confirmed using direct colony PCR with the primer pair of BCA-Dw Fw and BCA-Up Rv. The size of the PCR product from targeted transformants was about $2.1 \mathrm{~kb}$, which was shorter than those in wild type strains (about $5.0 \mathrm{~kb}$ ).

\section{Plasmids construction}

To construct plasmids for $p g s$-gene expression, the promoter region of the egl-S237 was amplified using PCR with genomic DNA from Bacillus sp. KSM-S237 as a template and the primer pair of P_S237 Fw and P_S237 Rv, and the amplified fragment was named P_S237. Similarly, pgs-gene fragments were PCR amplified using genomic DNA from Bacillus sp. KSM-366 and the primer pairs of Bsu pgsB Fw and Bsu pgsA Rv (PgsBCA), Bsu pgsB Fw and Bsu pgsC Rv (PgsBC), Bsu pgsC Fw and Bsu pgsA Rv (PgsCA), Bsu pgsB Fw and Bsu pgsB Rv (PgsB), Bsu pgsC Fw and Bsu pgsC Rv (PgsC), and Bsu pgsA Fw and Bsu pgsA Rv (PgsA). Subsequently, the fragments P_S237 and PgsBCA, PgsBC, PgsCA, PgsB, PgsC and PgsA were ligated using SOE-PCR to produce P_S237-PgsBCA, P_S237-PgsBC, P_S237-PgsB-PgsA, P_S237-PgsCA,

Table 1 Primers used for construction of mutants and plasmids

\begin{tabular}{|c|c|}
\hline Primer names & Sequence $\left(5^{\prime} \rightarrow 3^{\prime}\right)$ \\
\hline Cmr Fw & $\underline{\text { CAACTAAAGCACCCATTAGTTCAACAAACG }}$ \\
\hline Cmr Rv & CTTCAACTAACGGGGCAGGTTAGTGAC \\
\hline BCA-Dw Fw & CAAGCCCCGAGCAATCA \\
\hline BCA-Dw Rv & $\underline{\text { CTAATGGGTGCTTTAGTTGTCGGAGTGATAAAGATGAAATTTGTC }}$ \\
\hline BCA-Up Fw & $\underline{\text { CTGCCCCGTTAGTTGAAGTGCTTTTCGACATCTCCTT }}$ \\
\hline BCA-Up Rv & AAGGGTTTGTGATATCCGG \\
\hline P_S237 FW & CAACTAAAGCACCCATTAGGGATCCAACAGGCTTATATTTAGAG \\
\hline P_S237 Rv & $\underline{\text { CATCATATTACCTCCTAAATATTTTTTAAAGTA }}$ \\
\hline Bsu pgsa Rv & CGAAGCTTAGATGGCTTTGACAAATTTCATC \\
\hline Bsu pgsC Rv & CCCAAGCTTGACCTTCGGCGTTTCCGCT \\
\hline Bsu pgsB Rv & CCCAAGCTTGGCAGCGAATTTTCTGCGTCC \\
\hline Bsu pgsA Fw & ATTTAGGAGGTAATATGATGAAAAAAGAACTGAGCTTTCATG \\
\hline Bsu pgsC Fw & 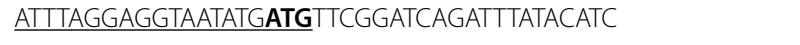 \\
\hline Bsu pgsB Fw & ATTTAGGAGGTAATATGATGTGGTTACTCATTATAGCCTG \\
\hline Bsu pgsBA Fw & CGTAAGCTAGGGGGAAATGCAGACGATGAAAAAAGAACTGAGCTTTCATG \\
\hline pHY-P_S237 Rv & CATCATATTACCTCCTAAATATTTTTAAAGTA \\
\hline pHY Fw & TAGAAGCTTGGGCAAAGCGTTTTTCCA \\
\hline Bam pgsB Fw & GGAGGTAATATGATGTGGTTACTCATTATAGCCTGTG \\
\hline Bli pgsB Fw & GGAGGTAATATGATGTGGGTAATGCTATTAGCCTGTG \\
\hline Obi pgsB Fw & GGAGGTAATATGATGCTAGAAGAATTTTTAATCATTT \\
\hline Bam pgsC Rv & TTGCCCAAGCTTCTACCTTCCCTGTCAGGATGCAG \\
\hline Bli pgsC Rv & TTGCCCAAGCTTCTACTTCCGACCCAAGTAAAGAC \\
\hline
\end{tabular}

Italic sequences represent synthesized restriction enzyme sites; underlined sequences represent homologous regions for spliced overlapping extension (SOE)-PCR; bold sequences represent initiation codons for the open reading frames (ORFs) of each gene 
P_S237-PgsB, P_S237-PgsC, and P_S237-PgsA. These ligated fragments were then digested with $\mathrm{BamHI}$ and HindIII and were introduced into the multi-cloning site of pHY300PLK. The resulting plasmids were named pHY-P_S237-PgsBCA, pHY-P_S237-PgsBC, pHY-P_ S237-PgsBA, pHY-P_S237-PgsCA, pHY-P_S237-PgsB, pHY-P_S237-PgsC, and pHY-P_S237-PgsA and were used as expression vectors for pgs-genes (Fig. 1).

\section{Cloning of homologous pgs-genes}

DNA fragments from the plasmid pHYS237, which digested with $X b a \mathrm{I}$ at multiple cloning sites of vector and open reading frame (ORF) of the egl-S237, were prepared as templates for PCR. DNA fragments as plasmid vector carrying the promoter region of egl-S237 were amplified using PCR with the primer pair of PHY-P_S237 $\mathrm{Rv}$ and $\mathrm{pHY}$ Fw. And then, the homologous pgs $B C$ of B. subtilis were also cloned using PCR. The pgsBC of $B$. amyloliquefaciens NBRC 3022 was amplified using PCR with genomic DNA as template and the primer pair of Bam pgsB Fw and Bam pgsC Rv, and the resulting fragment was named Bam-PgsBC. Similarly, the $p g s B C$ fragment from B. licheniformis ATCC 9945a was prepared using the primer pair of Bli pgsB Fw and Bli pgsC Rv (Bli$\mathrm{PgsBC}$ ), and the $p g s B C$ fragment from $O$. iheyensis JCM 11309 was prepared using the primer pair of Obi pgsB Fw and Obi pgsC Rv (Obi-PgsBC). Subsequently, directional cloning was performed using In-Fusion Cloning Kits with the DNA fragments pHY-P_S237 and Bam-PgsBC, pHY-P_S237 and Bli-PgsBC, and pHY-P_S237 and Obi-PgsBC. The constructed plasmids pHY-P_S237Bam-PgsBC, pHY-P_S237-Bli-PgsBC, and pHY-P_ S237-Obi-PgsBC were used as the expression vectors for heterologous pgs-genes.

\section{PGA production of transformants}

Expression vectors of $p g s B C$-genes and the control vector pHY300PLK were introduced using the protoplast transformation method (Chang and Cohen 1979). And then, the resulting transformants were selected on DM3Tc as single colonies, and were inoculated into media using an inoculating-loop. PGA production and cell growth were assessed as end-points using $\phi$ 25-mm culture tubes (NICHIDEN RIKA-GLASS Co., Ltd., Kobe, JPN) containing $5 \mathrm{~mL}$ of $2 \mathrm{xL} / \mathrm{Mal}$ and/or $2 \mathrm{xL} / \mathrm{Mal}+\mathrm{E} 8$ medium. Culture tubes were shaken using a reciprocal shaker HR-1000R (PRECi Co., Ltd., Tokyo, JPN) at $250 \mathrm{rpm}$ and $37^{\circ} \mathrm{C}$ for $72 \mathrm{~h}$. In addition, time course experiments using $500-\mathrm{mL}$ flask with baffles (Biott, Co., Tokyo, JPN) were examined, transformants on DM3Tc were pre-cultured in $\phi 25-\mathrm{mm}$ culture tubes containing $5 \mathrm{~mL}$ of LB supplemented with $15-\mathrm{ppm}$ tetracycline. After reciprocal shaking at $250 \mathrm{rpm}\left(30^{\circ} \mathrm{C}\right)$ for $20 \mathrm{~h}$ as pre-cultures, these broths were inoculated to an optical density at $600 \mathrm{~nm}$ $\left(\mathrm{OD}_{600}\right)$ of 0.1 in main cultures using shake-flasks containing $30 \mathrm{~mL}$ of $2 \mathrm{xL} / \mathrm{Mal}$ and/or $2 \mathrm{xL} / \mathrm{Mal}+\mathrm{E} 8$ medium. Subsequently, these flasks were shaken using a rotary shaker (TB-98R; PRECi Co., Ltd.) at $210 \mathrm{rpm}$ and $37^{\circ} \mathrm{C}$ for $72 \mathrm{~h}$. During this cultivation period, cell growth was periodically monitored by measuring $\mathrm{OD}_{600}$ using a UV2800A spectrophotometer (Hitachi High-technologies Co, Tokyo, JPN). If necessary, samples were diluted with $1 \%(\mathrm{w} / \mathrm{w}) \mathrm{NaCl}$ solution prior to determining $\mathrm{OD}_{600}$. Serial culture broths were also centrifuged at $14,000 \times g$ for $30 \mathrm{~min}$ at room temperature, and supernatants were then analyzed for PGA production using HPLC.

\section{PGA analyses}

PGA contents and molecular weights were determined using size exclusion chromatography. All samples were diluted with HPLC eluent, and were then filtered through $0.45-\mu \mathrm{m}$ Durapore membranes (Multiscreen MSHVN4510; Merck Millipore, Massachusetts, USA). Samples were then analyzed using HPLC with a tandem-jointed SEC column (TSKgel G6000PWXL + G4000PWXL; TOHSO, Tokyo, JPN). Samples were eluted with $0.1-\mathrm{M} \mathrm{Na}_{2} \mathrm{SO}_{4}$ at a flow rate of $1.0 \mathrm{~mL} / \mathrm{min}$ at $50{ }^{\circ} \mathrm{C}$, and eluted peaks were detected using a UV detector at a wavelength of $210 \mathrm{~nm}$. PGA contents were then calculated using a calibration curve that was prepared with food grade PGA additive (Meiji Food Materia, Tokyo, JPN), and the molecular weight of PGA was determined using the authentic pullulan STANDARD P-82 (Showa Denko, Tokyo, JPN). The stereo-chemical composition of PGA was determined as described previously (Ashiuchi et al. 2004; Ogawa et al. 1997). Produced PGA was then collected from culture broth using $\mathrm{EtOH}$ precipitation and was hydrolyzed with $6-\mathrm{N} \mathrm{HCl}$ at $105{ }^{\circ} \mathrm{C}$ for $15 \mathrm{~h}$. Total glutamic acid contents of hydrolyzed samples were measured using a L-8900 amino acid analyzer (Hitachi High-technologies Co, Tokyo, JPN), and L-glutamic acid contents were determined using L-glutamic acid assay kits (Kikkoman, Chiba, JPN). D-glutamic acid contents were calculated as the difference between total and L-glutamic acid contents, and D/L-ratios of PGA were then calculated.

\section{Sequence alignment and structure prediction}

Sequences were suitably aligned in multiple alignment analyses using the GENETYX program. Degrees of alignments were calculated using the "Multiple Alignment" program (Gap penalty; GAP Insert [-12], GAP Extend $[-4])$, and degrees of homology were assessed using the "Fasta Homology Search; fastp (protein-protein)" 
a

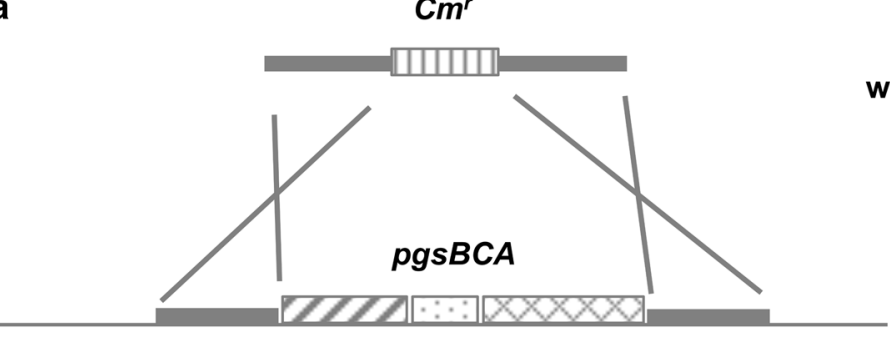

$\triangle$ pgs BCA -targeting DNA with the $\mathrm{Cm}$ resistant marker

Host strain genomic DNA (B. subtilis Marburg 168)

genome integration

pgsBCA-disrupted locus

(B. subtilis $\triangle 3$ genomic DNA)

b

pHY-P_S237-PgsBCA

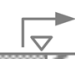

pHY-P_S237-PgsBC

pHY-P_S237-PgsBA

pHY-P_S237-PgsCA

pHY-P_S237-PgsB

pHY-P_S237-PgsC

pHY-P_S237-PgsA

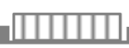

DNA fragment amplified by PCR (originated from Bacillus sp KSM-366)
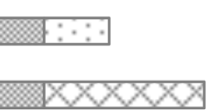

pHY-P_S237-Bam-PgsBC

pHY-P_S237-Bli-PgsBC

pHY-P_S237-Obi-PgsBC

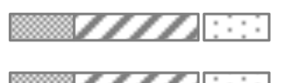

$\mathbb{1 2 7 2}: \because:$
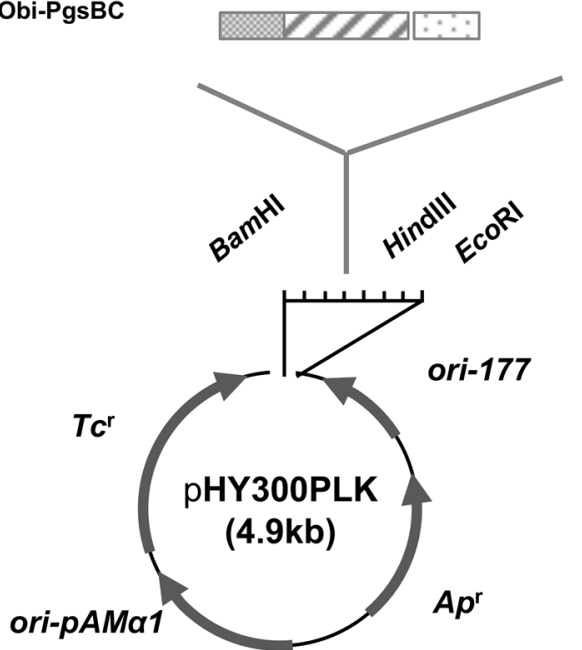

DNA fragment amplified by PCR

(originated from the heterologous Bacilli)

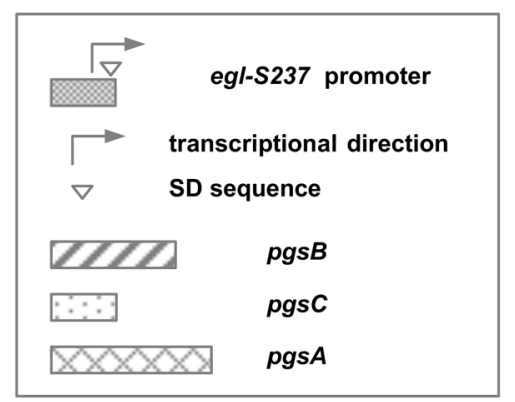

Fig. 1 Construction of pgsBCA deletion mutants and pgs-gene expression vectors. a Deletion of pgsBCA genes from B. subtilis Marburg No.168 by double crossover homologous recombination. $\mathbf{b}$ Construction of pgs-gene expression vectors. DNA fragments ( $p g s B C A-p g s A$ ) were amplified using spliced overlapping-extension (SOE)-PCR and were cloned into the plasmid pHY300PLK using restriction enzyme digestion. The heterologous Bacillus pgsBC fragments Bam-pgsBC, Bli-pgsBC, and Obi-pgsBC were then cloned using an in-fusion recombination cloning system 
program. Secondary structure predictions were made using the SOSUI system (SOSUI engine ver. 1.11; http:// harrier.nagahama-i-bio.ac.jp/sosui/) (Hirokawa et al. 1998).

\section{Nucleotide sequences and accession numbers}

Sequencing analyses showed that the cloned $p g s B$ from Bacillus sp. KSM-366 had an amino acid substitution (aspartate to glycine) at residue 27 (D27G) compared with the PgsB of B. subtilis Marburg 168 (BSORF ID, BG12531). Alternatively, the cloned pgsC from Bacillus sp. KSM-366 was identical to that of B. subtilis Marburg 168 (BSORF ID, BG12532). Further sequencing data of cloned pgsBC from B. amyloliquefaciens NBRC 3022 and B. lichenifromis ATCC 9945a gave similar results to those for $B$. amyloliquefaciens FZB42 (Genbank ID, ABS75634.1 and ABS75633.1) and B. licheniformis ATCC 14580 (GenBank ID, AAU25282.1 and AAU25281.1). Sequencing data for the cloned pgsBC of Bacillus sp. KSM-366, B. amyloliquefaciens NBRC 3022, and B. licheniformis ATCC 9945a were submitted to the DNA Data Bank of Japan (DDBJ; http://www.ddbj.nig. ac.jp). Sequencing data for each gene can be accessed using DDBJ accession numbers LC279207, LC279208, and LC279200.

\section{Results}

\section{PGA production by Bacillus transformants}

To identify genes that can be used for over-production of PGA in B. subtilis, we constructed seven pgs-gene expression vectors (Fig. 1b), and the transformed B. subtilis $\Delta 3$ strain with $p g s$-gene expression vectors using the protoplast method. Prepared transformants were cultivated in culture tubes with shaking for $72 \mathrm{~h}$ at $37^{\circ} \mathrm{C}$ in either $2 \mathrm{xL} /$ Mal medium or $2 \mathrm{xL} / \mathrm{Mal}+\mathrm{E} 8$ medium. $\mathrm{PGA}$ production by transformants was estimated using high-performance liquid chromatography (HPLC).
As shown in Table 2, both transformants with pHY-P S237-pgsBCA (P_BCA/A3) and pHY-P_S237-pgsBC ( $\mathrm{P} \_\mathrm{BC} / \triangle 3$ ) produced and secreted $\mathrm{PGA}$ into $2 \mathrm{xL} /$ $\mathrm{Mal}+\mathrm{E} 8$ medium, with yields of 3.0 and $26.0 \mathrm{~g} / \mathrm{L}$, respectively. The $\mathrm{P}_{-} \mathrm{BC} / \Delta 3$ strain also secreted PGA into $2 \mathrm{xL} /$ Mal medium with a final PGA yield of $4.0 \mathrm{~g} / \mathrm{L}$, whereas transformants harboring the pgs-gene expression vectors pHY-P_S237-pgsBA, pHY-P_S237-pgsCA, pHY-P_ S237-pgsB, pHY-P_S237-pgsC, pHY-P_S237-pgsA, and control vector pHY300PLK $\left(\mathrm{P}_{-} \mathrm{BA} / \Delta 3, \mathrm{P}_{-} \mathrm{CA} / \Delta 3\right.$, $\mathrm{P} \_\mathrm{B} / \Delta 3, \mathrm{P} \_\mathrm{C} / \Delta 3$, P_A $/ \Delta 3$, and $\mathrm{P} \_300 / \Delta 3$, respectively) did not produce PGA in $2 \mathrm{xL} / \mathrm{Mal}$ medium with or without L-glutamate.

In further experiments, cell growth was evaluated according to $\mathrm{OD}_{600}$ of culture broth. As shown in Table 2, growth of the $\mathrm{P} \_\mathrm{BC} / \Delta 3$ strain was substantially lower than that of the control strain P_300/ $\Delta 3$ in both media types, whereas growth of the other transformants were similar to control strains.

\section{Time course of PGA production by transformants}

To further investigate PGA production and cell growth of the $\mathrm{P} \_B C / \Delta 3$ strain, time course experiments were performed during batch fermentation in using $2 \mathrm{xL} / \mathrm{Mal}$ medium or $2 \times \mathrm{L} / \mathrm{Mal}+\mathrm{E} 8$ medium. As shown in Fig. 2, transformants exhibited triphasic growth curves in both media. Specifically, during the log-growth phase from 0 to $12 \mathrm{~h}, \mathrm{OD}_{600}$ values increased exponentially from 0.1 to more than 10 in both media. Subsequently, a late loggrowth phase was observed until the $36 \mathrm{~h}$ time point, with modest increases in $\mathrm{OD}_{600}$ values, and a stationary phase was observed thereafter with no increase in $\mathrm{OD}_{600}$. In comparisons of $\mathrm{P} \_\mathrm{BC} / \Delta 3$ and $\mathrm{P} \_300 / \Delta 3$ (control strain), cell growth of the $\mathrm{P}_{-} \mathrm{BC} / \Delta 3$ strain was decreased to $60-70 \%$ of the control strains in $2 \times \mathrm{L} / \mathrm{Mal}$ medium, and was decreased to $50-60 \%$ of the control strains in $2 \times \mathrm{L} /$ Mal+E8 medium.

Table 2 Growth and PGA production by Bacillus transformants

\begin{tabular}{|c|c|c|c|c|c|c|}
\hline \multirow[t]{2}{*}{ Strain } & \multicolumn{3}{|l|}{ 2xL/Mal } & \multicolumn{3}{|c|}{ 2xL/Mal+E8 } \\
\hline & $O D_{600}$ & $\mathrm{Mw} / 10^{3}$ & $\operatorname{Prod}(g / L)$ & $\mathrm{OD}_{600}$ & $\mathrm{Mw} / 10^{3}$ & Prod (g/L) \\
\hline P_300/43 & $34 \pm 3.3$ & nd & nd & $47 \pm 1.6$ & nd & nd \\
\hline $\mathrm{P} \_\mathrm{BCA} / \triangle 3$ & $34 \pm 6.2$ & nd & nd & $40 \pm 10$ & $1000 \pm 200$ & $3.0 \pm 0.7$ \\
\hline P_BC/ $\triangle 3$ & $14 \pm 4.1$ & $400 \pm 100$ & $4.0 \pm 0.3$ & $16 \pm 4.6$ & $1100 \pm 200$ & $26.0 \pm 3.1$ \\
\hline P_BA/ $\triangle 3$ & $38 \pm 2.7$ & nd & nd & $30 \pm 2.8$ & nd & nd \\
\hline P_CA/ $\triangle 3$ & $38 \pm 1.0$ & nd & nd & $41 \pm 3.2$ & nd & nd \\
\hline$P \_B / \Delta 3$ & $33 \pm 3.8$ & nd & nd & $27 \pm 2.4$ & nd & nd \\
\hline $\mathrm{P} \_\mathrm{C} / \Delta 3$ & $34 \pm 1.6$ & nd & nd & $40 \pm 2.3$ & nd & nd \\
\hline P_A/ $\triangle 3$ & $37 \pm 1.5$ & nd & nd & $47 \pm 2.3$ & nd & nd \\
\hline
\end{tabular}

Data are presented as means of five independent experiments $\pm S E(n=5)$ 

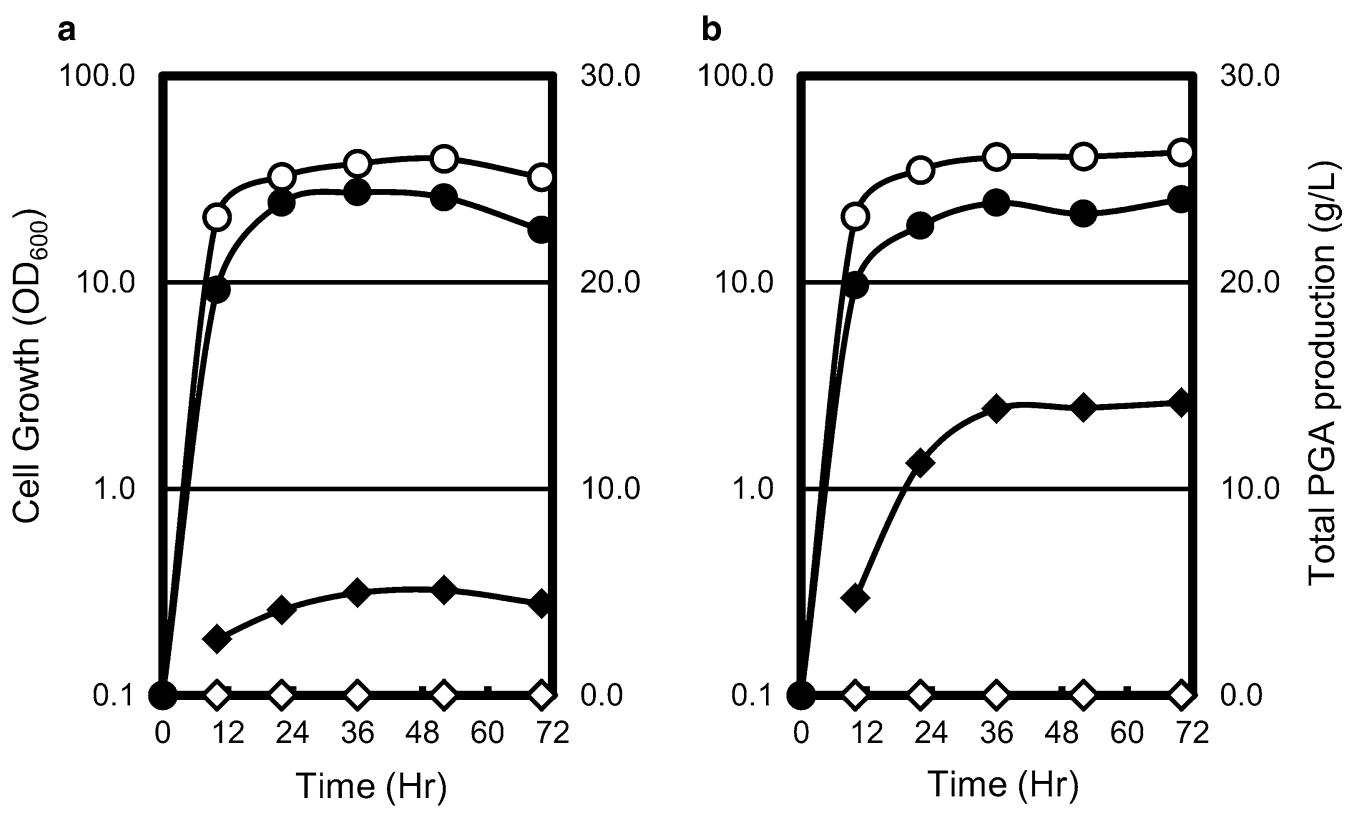

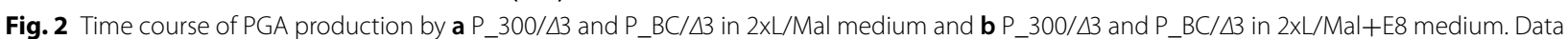
are presented as means of five independent experiments. Symbols: opened circles, cell growth of P_300/ $\Delta 3$; opened diamonds, PGA production by P_300/ $\boldsymbol{\Delta} 3$; closed circles, cell growth of P_BC/ $\boldsymbol{\Delta}$; ; closed diamonds, PGA production by $\mathrm{P} \_\mathrm{BC} \boldsymbol{\Delta} 3$

PGA production was detectable at $10 \mathrm{~h}$ after inoculation of cultures, and reached the maximum level at $36 \mathrm{~h}$. Maximum PGA production reached $5 \mathrm{~g} / \mathrm{L}$ $\left(\mathrm{Mw} / 10^{3}=2600\right)$ in $2 \mathrm{xL} / \mathrm{Mal}$ medium and $14 \mathrm{~g} / \mathrm{L}$ $\left(\mathrm{Mw} / 10^{3}=2100\right)$ in $2 \mathrm{xL} / \mathrm{Mal}+\mathrm{E} 8$ medium. Furthermore, PGA contents of culture broth were maintained at the maximum level after $36 \mathrm{~h}$, and the molecular weights of PGA were maintained during the $72 \mathrm{~h}$ cultivation.

Amino acid sequence alignment and structural estimation To characterize the PGA synthetase complex, we performed amino acid sequence alignments using the GENETYX program and determined homology between PgsBC proteins. As shown in Fig. 3, all of the present PgsB had the conserved sequence GIRGKS (residues 37-42 of Bsu-PgsB), which was presumed to be the ATP-binding motif (Aboulmagd et al. 2000; Ashiuchi et al. 2001). Furthermore, the in-phase initiation codon (Urushibata et al. 2002) of the overlapping gene (97Met of Bsu-PgsB) was conserved in present proteins but not in Ban-PgsB and Obi-PgsB.

Sequence homology was determined between PgsB from B. subtilis Marburg 168 (Bsu) and the other heterologous PgsB, and matching scores were 92\% for PgsB from B. amyloliquefaciens NBRC 3022 (Bam-PgsB), 89\% for B. licheniformis ATCC 9945a (Bli-PgsB), 67\% for $B$. anthracis TE702 (Ban-PgsB), and 55\% for Oceanobacillus iheyensis JCM 11309 (Obi-PgsB). Similarly, homology searches for PgsC gave matching scores of 93\% for the PgsC from B. amyloliquefaciens NBRC 3022 (Bam-PgsC), 89\% for B. licheniformis ATCC 9945a (Bli-PgsC), 77\% for B. anthracis TE702 (Ban-PgsC), and 51\% for Oceanobacillus iheyensis JCM 11309 (Obi-PgsC).

In structure estimations of PgsB and PgsC using the SOSUI program (ver. 1.11) as shown in Fig. 3, all PgsB except Bli-PgsB had hydrophobic moieties (residues 1-23 of Bsu-PgsB) in N-terminal regions, which comprise transmembrane helixes. The structure of PgsC was also predicted using the SOSUI program, which indicated the presence of five transmembrane helices (residues 1-23, 25-47, 51-72, 77-99, and 125-147 of Bsu-PgsC), one intracellular loop (residues 73-87 of Bsu-PgsC), and one extracellular loop (residues 111125 of Bsu-PgsC).

\section{PGA production by transformants harboring heterologous}

\section{pgsBC genes}

To investigate the potential for over-production of PGA in B. subtilis expressing heterologous PgsBC, $B$. subtilis $\Delta 3$ strains were transformed with a series of heterologous pgs-gene expression vectors ( $\mathrm{pHY}-\mathrm{P}_{-}$ S237-Bam-pgsBC, pHY-P_S237-Bli-pgsBC, and pHY-P_S237-Obi-pgsBC; Fig. 1a). The prepared transformants were then cultivated in shaking flasks containing $2 x \mathrm{~L} / \mathrm{Mal}+\mathrm{E} 8$ medium for $72 \mathrm{~h}$ at $37{ }^{\circ} \mathrm{C}$. The 

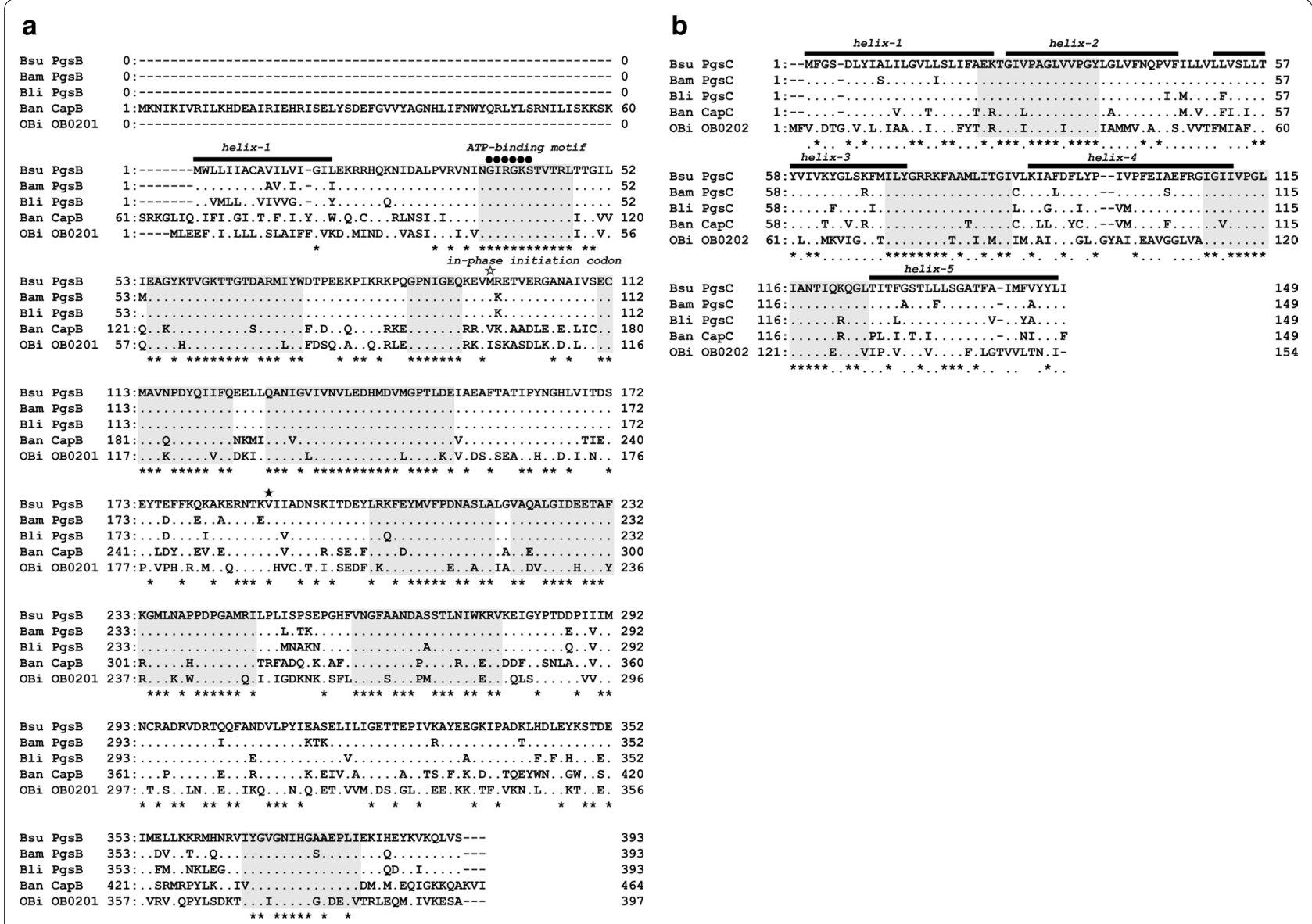

Fig. 3 Multiple alignments of a PgsB and $\mathbf{b}$ PgsC proteins. Sequence alignments were performed using the GENETYX program. Identical residues are shown by periods (.) and sites with $100 \%$ homology are marked with asterisks $\left(^{*}\right)$ on the bottom of the line. Predicted consensus sequences are shaded in Gray tones. Secondary structure prediction was performed using the SOSUI program. Predicted transmembrane helix structures are shown as black lines on the tops of lines. Symbols: closed circles, ATP-binding motif; opened star, the initiation codon of in-phase overlapping pgsB genes of B. subtilis; closed star, the initiation codon of in-phase overlapping capB genes of $B$. anthracis

transformants harboring pHY-P_S237-Bam-pgsBC (P_Bam-BC/ $\Delta 3$ ), pHY-P_S237-Bli-pgsBC (P_Bli$\mathrm{BC} / \Delta 3)$, and $\mathrm{pHY}-\mathrm{P} \_\mathrm{S} 237-\mathrm{Obi}-\mathrm{pgsBC}\left(\mathrm{P} \_\mathrm{Obi}-\mathrm{BC} / \Delta 3\right)$ produced 3.3-, 1.4-, and 0.7-g PGA/L at molecular weights of $1100 \mathrm{k}, 2700 \mathrm{k}$, and $3300 \mathrm{k}$, respectively.

\section{Identification of stereo-chemical compositions}

To assess $\mathrm{D} / \mathrm{L}$-ratios of glutamate in produced PGA, polymers were isolated from culture broth samples and were acid-hydrolyzed as described above. As shown in Table 3, the $\mathrm{D} / \mathrm{L}$-ratio of PGA from $\mathrm{P} \_\mathrm{BCA} / \Delta 3$ transformants, which harbored all components of the PgsBCA complex, was $75 / 25$, and this value is close to that of PGA in the Japanese traditional food Natto (Ashiuchi 2013; Nagai et al. 1997). In contrast, the D/L-ratio of PGA from the $\mathrm{P} \_\mathrm{BC} / \Delta 3$ strain was $4 / 96$, indicating a marked increase in L-glutamic acid. Furthermore, PGA from P_Bam-BC/ $\Delta 3$,
Table 3 Chirality of PGA from transformants

\begin{tabular}{|c|c|c|c|c|c|}
\hline Strain & Medium $^{a}$ & $O D_{600}$ & $\mathrm{Mw} / 10^{3}$ & Prod (g/L) & D/L-ratio \\
\hline $\mathrm{P} \_\mathrm{BC} / \triangle 3$ & L-Glu & 29.6 & 2000 & 35.3 & $4 / 96$ \\
\hline 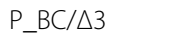 & D-Glu & 17.5 & 600 & 3.1 & $4 / 96$ \\
\hline P_Bam-BC/ $\triangle 3$ & L-Glu & 37.9 & 1100 & 2.5 & $4 / 96$ \\
\hline P_Bli-BC/ $\triangle 3$ & L-Glu & 36.4 & 2800 & 1.4 & $2 / 98$ \\
\hline P_Obi-BC/ $\triangle 3$ & L-Glu & 39.0 & 1900 & $0.7^{b}$ & $11 / 89$ \\
\hline
\end{tabular}

${ }^{a}$ Medium; L-Glu was used 2xL/Mal+E8 (which contained $0.43 \mathrm{M} \mathrm{L-glutamic} \mathrm{acid)}$ and D-Glu was used $2 \times \mathrm{L} / \mathrm{Mal}$, containing $0.43 \mathrm{M} \mathrm{D}$-glutamic acid and adjusted to the same $\mathrm{pH}$ as $2 \times \mathrm{L} / \mathrm{Mal}+\mathrm{E} 8$ with $\mathrm{NaOH}$. Data are presented as means of five independent experiments

b PGA production of $\mathrm{P}_{-} \mathrm{Obi}-\mathrm{BC} / \Delta 3$ strains is representative of three-fifth or twofifth of positive transformants (it seems to be unstable)

P_Bli-BC/ $\Delta 3$, and P_Obi-BC/ $\Delta 3$ transformants had D/Lratios of $4 / 96,2 / 98$, and $11 / 89$, respectively. 


\section{Discussion}

PGA is synthesized and secreted by several Bacillus strains (Ashiuchi 2013; Luo et al. 2016). In previous studies, various experimental maneuvers were applied to elucidate PGA synthesis. Makino et al. first reported that the CapBCA of $B$. anthracis, which are membrane-associated proteins, catalyzes the synthesis of D-PGA (Makino et al. 1989). Subsequently, Ashiuchi et al. cloned B. subtilis NBRC 3336 pgs $B C A$, which are homologous to the cap $B C A$ of $B$. anthracis, were characterized these genes as the membrane-associated PGA synthetases (Ashiuchi et al. 1999, 2001). In these studies, they concluded that all pgsBCA are essential genes for PGA synthesis. On the other hand, Urushibata et al. cloned the $y w s C$ (homolog of pgsB) of B. subtilis NBRC 16449 (Urushibata et al. 2002), and demonstrated that the histidine-tagged YwsC solely synthesized PGA. They concluded that both YwsC and YwtA (homolog of PgsC) are essential for PGA synthesis, and that YwtB (homolog of PgsA) seems to be required for maximum PGA production.

To identify the components responsible for over-production of PGA, we used the vectors that allowed highlevel expression of genes and the B. subtilis mutant strain $(\Delta 3)$. Our experimental data indicated that the transformants $P_{-} B C A / \Delta 3$ and $P_{-} B C / \Delta 3$ produced $P G A$, indicating that PgsA is not essential for PGA production. In addition, transformants harboring either pHY-P_S237PgsCA or pHY-P_S237-PgsC were unable to produce PGA, indicating that PgsB is essential for PGA production. Furthermore, transformants with the vectors $\mathrm{pHY}-\mathrm{P}_{-}$ S237-PgsBA or pHY-P_S237-PgsB did not lead to PGA production, further confirming the requirement of $\mathrm{PgsC}$ for PGA production. In our present studies, B. subtilis having no pgsA produced abundant PGA and the produced PGA exhibited an L-rich enantiomeric ratio. These data are partially in agreement with those reported by Ashiuchi et al. who indicated glutamate-dependent ATPase activity of both PgsBCA and PgsBC (Ashiuchi et al. 2001). On the other hands, our findings contradict those showed by Ashiuchi et al. who indicated the inability of PGA production using B. subtilis MA41 strains (genomic pgsBCA-gene disruptants) and pWPGBC1, which D-xylose inducible gene expression vector for $p g s B C$ (Ashiuchi et al. 2006). We speculated that there are differences between the substance-inducible promoter and the constitutive highlevel expression promoter. Additionally, our findings were not consistent with the results by Urushibata et al. who exhibited that the purified histidine-tagged PgsB synthesized PGA from L-glutamate in vitro system (Urushibata et al. 2002). In the view of these experimental facts, there was a possibility that the high sensitivity analysis using radioactive-labeled substances detected PGA synthesis by only PgsB. From the viewpoint of nutrient metabolism,
PGA production seemed to negatively influence over host cell-growth. Actually, the maximum $\mathrm{OD}_{600}$ value of the P_PgsBC/ $\Delta 3$ strain drastically decreased to that of control strains. In addition, the yield of PGA of P_PgsBC/ $\Delta 3$ strain in $2 \times \mathrm{L} / \mathrm{Mal}$ medium was higher than the initial glutamic acid content. These data indicated that other amino acids in the growth medium were utilized for PGA synthesis, and cell growth was reduced as a consequent. Moreover the P_PgsBCA/ $\Delta 3$ strains did not produce PGA in batch fermentation with shaking flasks (data not shown). These results suggested that over-expression of $p g s B C A$ conceivably caused in stress like nitrogen starvation as D-glutamate auxotrophy (Ashiuchi et al. 2007). Also, as previously reported, PGA production was improved by NADPH regeneration in B. licheniformis WX-02 (Cai et al. 2017). Taken together, these data warrant speculation that PGA synthesis in vivo system seems like to be influenced by not only synthesis machinery but also nitrogen and energy metabolism.

In this study, we confirmed PGA production by the transformants harboring heterologous PgsBC. And then, these sequence homology and structure were verified. Although the homology was low between Bsu-PgsB and Obi-PgsB (55\%) and between Bsu-PgsC and Obi-PgsC (51\%), transformants harboring Obi-PgsBC produced PGA. Accordingly, structural estimations using the SOSUI program indicated that PgsB homologs all have one transmembrane helix, whereas PgsC homologs have five transmembrane helices. Moreover, predicted consensus regions were found in both PgsB and PgsC, and two conserved regions of $\mathrm{PgsC}$ were located in the loop structure. These results indicate that similarities in protein structures are more important than similarities in amino acid sequences. Similar interpretations were suggested in the ADP-forming amide bond ligase superfamily (Sheng et al. 2000; Urushibata et al. 2002). Assessments of $\mathrm{D} / \mathrm{L}-$ ratios of produced PGAs showed that $\mathrm{P} \_B C A_{-} \Delta 3$ produced DL-PGA, whereas P_BC/ $\triangle 3$ produced L-PGA. Hence, L-PGA can be synthesized in the absence of PgsA, suggesting interactions between PgsA and racemase and/ or conformational changes induced by binding of PgsA to PgsBC complex (Sheng et al. 2000).

In a previous study, Urushibata et al. showed that YwtA (homolog of $\mathrm{PgsC}$ ) is a hydrophobic cell membrane protein that transports PGA in B. subtilis (Urushibata et al. 2002). Ashiuchi et al. suggested that PgsB and PgsC are naturally associated with the cell membrane and have glutamate-dependent ATPase activity (Ashiuchi et al. 2001). Our present data using growing cells suggest that the PgsBC complex is critical for excretion of excess PGA and that this over-production system causes stress to host cells. Thus, to further improve PGA production, studies will be designed to characterize interactions of 
PgsBC with cell membranes, and to resolve the competition between PGA production and cell growth so that glutamate, ATP, and NADPH can be optimally provided.

In conclusion, the present study shows that PgsBC is essential for over-production of PGA. Additionally, we developed a promising method for modifying $\mathrm{D} / \mathrm{L}$-ratios of PGA in favor of the useful material L-PGA (Yamasaki et al. 2010).

\begin{abstract}
Abbreviations
$P G A$ : poly-gamma-glutamic acid; $D / L$-ratio: enantiomeric ratio of D- and L-glutamic acid; $k D a$ : kilo Dalton; $C m r$ : chloramphenicol-resistant gene; LB: Luria-Bertani; $L B C m$ : $L B$ agar plates containing 10-ppm chloramphenicol; DM3TC: DM3 media containing 30-ppm tetracycline; LBTC: LB agar plates containing 15-ppm tetracycline; ORF: open reading frame; $O D_{600}$ : optical density at $600 \mathrm{~nm}$.
\end{abstract}

\section{Authors' contributions}

All authors have contributed to this research work. All authors read and approved the final manuscript.

\section{Author details \\ ${ }^{1}$ Global R\&D-Biological Science Research, Kao Corporation, 2606 Akabane, Ichikai, Haga, Tochigi 321-3497, Japan. ${ }^{2}$ Global R\&D-Safety Science Research, Kao Corporation, 2606 Akabane, Ichikai, Haga, Tochigi 321-3497, Japan. \\ ${ }^{3}$ Global R\&D-Strategy and Planning, Kao Corporation, 2-1-3 Bunnka, Sumida, Tokyo 131-8501, Japan.}

\section{Acknowledgements}

We thank Reiko Shimooka and Kaori Matsumoto for their experimental assistance. We also thank Yoshinori Takema and Tadashi Hase (Kao Corporation) for valuable advice. This study was implemented in collaboration with Seiichi Miyanaga, Tadashi Igarashi, Kenichi Shiba, and Kenichi Shikata (Kao Corporation). Finally, we thank Shenghao Liu (Kao Corporation) for helpful communications and useful comments on this manuscript and experiments. We would like to also thank Enago (http://www.enago.jp) for the English language review.

\section{Competing interests}

The authors declare that they have no competing interests.

\section{Availability of data and materials}

All data supporting the conclusions are presented in the main article.

\section{Consent for publication}

Not applicable.

\section{Ethics approval and consent to participate}

Not applicable. This article does not contain any experiments with human participants or animals performed by any of the authors.

\section{Funding}

Not applicable.

\section{Publisher's Note}

Springer Nature remains neutral with regard to jurisdictional claims in published maps and institutional affiliations.

Received: 16 June 2018 Accepted: 21 June 2018

Published online: 03 July 2018

\section{References}

Aboulmagd E, Oppermann-Sanio FB, Steinbüchel A (2000) Molecular characterization of the cyanophycin synthetase from Synechocystis sp. strain PCC6308. Arch Microbiol 174(5):297-306

Ashiuchi M (2013) Microbial production and chemical transformation of poly-gamma-glutamate. Microb Biotechnol 6(6):664-674. https://doi. org/10.1111/1751-7915.12072

Ashiuchi M, Misono H (2002) Biochemistry and molecular genetics of polygamma-glutamate synthesis. Appl Microbiol Biotechnol 59(1):9-14. https ://doi.org/10.1007/s00253-002-0984-x

Ashiuchi M, Misono Y (2005) poly-gamma-glutamic acid. In: Steinbüchel A, Doi $Y$ (eds) Biotechnology of biopolymers from synthesis to patents. Wiley, Weinheim, pp 791-841

Ashiuchi M, Soda K, Misono H (1999) A poly-gamma-glutamate synthetic system of Bacillus subtilis IFO 3336: gene cloning and biochemical analysis of poly-gamma-glutamate produced by Escherichia coli clone cells. Biochem Biophys Res Commun 263(1):6-12. https://doi.org/10.1006/ bbrc.1999.1298

Ashiuchi M, Nawa C, Kamei T, Song JJ, Hong SP, Sung MH, Soda K, Misono H (2001) Physiological and biochemical characteristics of poly gammaglutamate synthetase complex of Bacillus subtilis. Eur J Biochem 268(20):5321-5328

Ashiuchi M, Shimanouchi K, Nakamura H, Kamei T, Soda K, Park C, Sung MH, Misono H (2004) Enzymatic synthesis of high-molecular-mass poly-gamma-glutamate and regulation of its stereochemistry. Appl Environ Microbiol 70(7):4249-4255. https://doi.org/10.1128/ aem.70.7.4249-4255.2004

Ashiuchi M, Shimanouchi K, Horiuchi T, Kamei T, Misono H (2006) Genetically engineered poly-gamma-glutamate producer from Bacillus subtilis ISW1214. Biosci Biotechnol Biochem 70(7):1794-1797. https://doi. org/10.1271/bbb.60082

Ashiuchi M, Nishikawa Y, Ki Matsunaga, Yamamoto M, Shimanouchi K, Misono H (2007) Genetic design of conditional D-glutamate auxotrophy for Bacillus subtilis: use of a vector-borne poly- $\gamma$-glutamate synthetic system. Biochem Biophys Res Commun 362(3):646-650

Buescher JM, Margaritis A (2007) Microbial biosynthesis of polyglutamic acid biopolymer and applications in the biopharmaceutical, biomedical and food industries. Crit Rev Biotechnol 27(1):1-19. https://doi. org/10.1080/07388550601166458

Cai D, He P, Lu X, Zhu C, Zhu J, Zhan Y, Wang Q, Wen Z, Chen S (2017) A novel approach to improve poly-gamma-glutamic acid production by NADPH Regeneration in Bacillus licheniformis WX-02. Sci Rep 7:43404. https://doi. org/10.1038/srep43404

Chang S, Cohen SN (1979) High frequency transformation of Bacillus subtilis protoplasts by plasmid DNA. Mol Gen Genet 168(1):111-115. https://doi. org/10.1007/BF00267940

Ehrlich SD (1977) Replication and expression of plasmids from Staphylococcus aureus in Bacillus subtilis. Proc Natl Acad Sci USA 74(4):1680-1682

Hakamada Y, Hatada Y, Koike K, Yoshimatsu T, Kawai S, Kobayashi T, Ito S (2000) Deduced amino acid sequence and possible catalytic residues of a thermostable, alkaline cellulase from an Alkaliphilic Bacillus strain. Biosci Biotechnol Biochem 64(11):2281-2289

Hara T, Nakajima K, Saito H, Ishizaki A, Ogata S, Ueda S (1992) Sequence analysis of replication origin of plasmid pLS11 of Bacillus subtilis IFO 3022. Biosci Biotechnol Biochem 56(2):223-227

Hirokawa T, Boon-Chieng S, Mitaku S (1998) SOSUl: classification and secondary structure prediction system for membrane proteins. Bioinformatics 14(4):378-379

Horton RM, Hunt HD, Ho SN, Pullen JK, Pease LR (1989) Engineering hybrid genes without the use of restriction enzymes: gene splicing by overlap extension. Gene 77(1):61-68

Leenhouts K, Buist G, Kok J (1999) Anchoring of proteins to lactic acid bacteria. Antonie Van Leeuwenhoek 76(1-4):367-376

Luo Z, Guo Y, Liu J, Qiu H, Zhao M, Zou W, Li S (2016) Microbial synthesis of poly-gamma-glutamic acid: current progress, challenges, and future perspectives. Biotechnol Biofuels 9:134. https://doi.org/10.1186/s1306 8-016-0537-7

Makino S, Uchida I, Terakado N, Sasakawa C, Yoshikawa M (1989) Molecular characterization and protein analysis of the cap region, which is essential for encapsulation in Bacillus anthracis. J Bacteriol 171(2):722-730 
Manabe K, Kageyama Y, Morimoto T, Ozawa T, Sawada K, Endo K, Tohata M, Ara K, Ozaki K, Ogasawara N (2011) Combined effect of improved cell yield and increased specific productivity enhances recombinant enzyme production in genome-reduced Bacillus subtilis strain MGB874. Appl Environ Microbiol 77(23):8370-8381. https://doi.org/10.1128/AEM.06136-11

Nagai T, Koguchi K, Itoh Y (1997) Chemical analysis of poly-gamma-glutamic acid produced by plasmid-free Bacillus subtilis (natto): evidence that plasmids are not involved in poly-gamma-glutamic acid production. J Gen Appl Microbiol 43(3):139-143

Narita J, Okano K, Kitao T, Ishida S, Sewaki T, Sung MH, Fukuda H, Kondo A (2006) Display of alpha-amylase on the surface of Lactobacillus casei cells by use of the PgsA anchor protein, and production of lactic acid from starch. Appl Environ Microbiol 72(1):269-275. https://doi.org/10.1128/ aem.72.1.269-275.2006

Ogawa Y, Yamaguchi F, Yuasa K, Tahara Y (1997) Efficient production of Y-polyglutamic acid by Bacillus subtilis (natto) in jar fermenters. Biosci Biotechnol Biochem 61(10):1684-1687

Potter M, Oppermann-Sanio FB, Steinbüchel A (2001) Cultivation of bacteria producing polyamino acids with liquid manure as carbon and nitrogen source. Appl Environ Microbiol 67(2):617-622. https://doi.org/10.1128/ aem.67.2.617-622.2001

Saito H, Miura KI (1963) Preparation of transforming deoxyribonucleic acid by phenol treatment. Biochim Biophys Acta 72:619-629
Saito T, Iso N, Mizuno H, Kaneda H, Suyama Y, Kawamura S, Osawa S (1974) Conformational change of a Natto mucin in solution. Agric Biol Chem 38(10):1941-1946. https://doi.org/10.1271/bbb1961.38.1941

Sheng Y, Sun X, Shen Y, Bognar AL, Baker EN, Smith CA (2000) Structural and functional similarities in the ADP-forming amide bond ligase superfamily: implications for a substrate-induced conformational change in folylpolyglutamate synthetase. J Mol Biol 302(2):425-438. https://doi.org/10.1006/ jmbi.2000.3987

Takami H, Takaki Y, Uchiyama I (2002) Genome sequence of Oceanobacillus iheyensis isolated from the Iheya Ridge and its unexpected adaptive capabilities to extreme environments. Nucleic Acids Res 30(18):3927-3935

Urushibata Y, Tokuyama S, Tahara Y (2002) Characterization of the Bacillus subtilis yws $C$ gene, involved in gamma-polyglutamic acid production. J Bacteriol 184(2):337-343

Yamasaki D, Minouchi Y, Ashiuchi M (2010) Extremolyte-like applicability of an archaeal exopolymer, poly-gamma-L-glutamate. Environ Technol 31(10):1129-1134. https://doi.org/10.1080/09593331003592279

Young FE, Spizizen J (1963) Incorporation of deoxyribonucleic acid in the Bacillus subtilis transformation system. J Bacteriol 86:392-400

\section{Submit your manuscript to a SpringerOpen ${ }^{\odot}$ journal and benefit from:}

- Convenient online submission

- Rigorous peer review

- Open access: articles freely available online

- High visibility within the field

- Retaining the copyright to your article

Submit your next manuscript at $\boldsymbol{\nabla}$ springeropen.com 